Research Article

\title{
The Analysis and Modeling of the Synthetical Meshing Stiffness of Inner Gearing considering the Flexible Inner Ring Gear
}

\author{
Shengyang Hu $(\mathbb{D}$ and Zongde Fang \\ School of Mechanical Engineering, Northwestern Polytechnical University, Xi'an 710072, China \\ Correspondence should be addressed to Shengyang Hu; hushengyangnwpu@163.com
}

Received 22 December 2018; Accepted 4 April 2019; Published 23 April 2019

Academic Editor: Chengzhi Shi

Copyright (C) 2019 Shengyang Hu and Zongde Fang. This is an open access article distributed under the Creative Commons Attribution License, which permits unrestricted use, distribution, and reproduction in any medium, provided the original work is properly cited.

\begin{abstract}
As a key part of vibration generation and transmission of planetary gear transmissions, thin-walled inner ring gear deforms under the influence of meshing excitation and seriously affects the reliability and fatigue life of the transmission system. The effect of the flexibility of the inner ring gear on the transmission system is ignored in the calculation of making the inner ring gear as a rigid body in the lumped parameter model, while the calculation amount of the finite element model is too large. Therefore, it is very important to establish an accurate and reasonable model to solve the flexibility of the inner ring gear. In this paper, according to the supporting mode, supporting quantity, thickness, and sectional shape of the inner ring gear, the inner ring gear is reasonably separated into the form of multisection curved beam. The displacement of the gear teeth in the meshing line caused by the flexibility of the inner ring gear is obtained rapidly and accurately. It lays an important theoretical foundation for the dynamic analysis of planetary gear transmission.
\end{abstract}

\section{Introduction}

The inner ring gear is a key component for the generation and transmission of planetary gear system vibration. In order to reduce the transmission weight and reduce the system vibration and noise, the inner ring gear $[1,2]$ and the elastic connection mode [3] are mostly adopted in the planetary gear transmission system to prevent the vibration of the planetary gear transmission system from being transmitted to the body. The vibration behavior of thinwalled inner ring gear is very complicated due to the complex working condition and the influence of meshing excitation, so its reliability and fatigue life are seriously affected. Therefore, it is of great significance to analyze the flexibility of inner ring gear to reveal the dynamic performance of the system and to design a planetary transmission device with light, low vibration, and high reliability. Some scholars [4-7] used the lumped mass method to establish the dynamic model of the spur gear planetary gear system, which included the flexibility of inner ring gear, and analyzed its inherent characteristics. Chen's $[8,9]$ theoretical model of nonlinear excitation of meshing stiffness about flexible ring gear reveals the deformation of inner ring gear under nonlinear large deformation. However, the solution model of the elastic deformation of the inner ring gear under linear conditions is not given.

Through analyzing the existing lumped parameter model and finite element model, the lumped parameter model fails to take into account the elastic deformation of transmission components, especially the flexible inner ring gear, so the analysis accuracy still needs to be improved. In contrast, the finite element model can accurately reflect the load condition of the planetary transmission system by taking into account the elastic deformation of each component. However, the disadvantage of this model is that the modeling process is complex, the degree of freedom of the model is large, and the solution is time-consuming. Therefore, in this paper, the inner ring gear is considered to 
be composed of matrix and gear teeth. The overall meshing stiffness of the inner gearing teeth is obtained by LTCA, while the flexural deformation of the inner ring gear is simulated by the curved beam model to obtain the amount of displacement generated by normal meshing force and the rigidity on the meshing line caused by the flexibility of the inner ring gear. Because the curved beam is close to the flexible inner ring gear on the geometric model, it can reduce the number of sections and calculation and is more accurate than the straight beam model. By analyzing the different supporting modes of the flexibility of inner ring gear and combining with the boundary conditions of the corresponding curved beam, the corresponding formulas of bending, bending angle, and torsion angle are derived, and thus, the comprehensive deformation displacement of the meshing line of the flexible inner ring gear due to torsional angle deformation, bending deformation, and shear deformation is obtained. This model provides an important basis for accurate dynamic analysis of the planetary transmission system.

\section{Analysis of Flexible Deformation of Inner Ring Gear}

This paper mainly studies the calculation method of inner ring gear deformation under two supporting modes (fixed support and pin support). Assuming that the inner ring gear has $n$ supports, the inner ring gear can be divided into curved beams [10] that are uniformly curved at least $n$ segments (Figure 1).

Setting of boundary conditions for supporting mode:

(1) Bolt connection is usually used for the inner ring gear with fixed support. The translational displacement of the beam at the support position is completely constrained, and only the rotational degree of freedom is retained, that is, $u=0 ; \omega=$ $0 ; \theta_{1}=\theta_{2}$.

(2) There are two kinds of boundary conditions for the pin supported inner ring gear. When the beam is far away from the center of the circle along the radial direction, it will be in contact with the pin. The circumferential displacement and radial displacement of the beam at the support position are completely restrained. At this point, the boundary condition is the same as the fixed mode, namely, $u=0 ; \omega=0 ; \theta_{1}=\theta_{2}$. When the beam moves along the radial direction close to the center of the circle, it will be separated by contact with the pin. The circumferential displacement and radial displacement of the beam at the support position can be carried out simultaneously. At this point, the boundary condition is $u_{1}=u_{2} ; \omega_{1}=$ $\omega_{2} ; \theta_{1}=\theta_{2}$.

Based on the above analysis, the corresponding deformation, internal force, and torque can be obtained by setting different boundary conditions for arbitrary boundary constraint positions of any section of the curved beam, thus

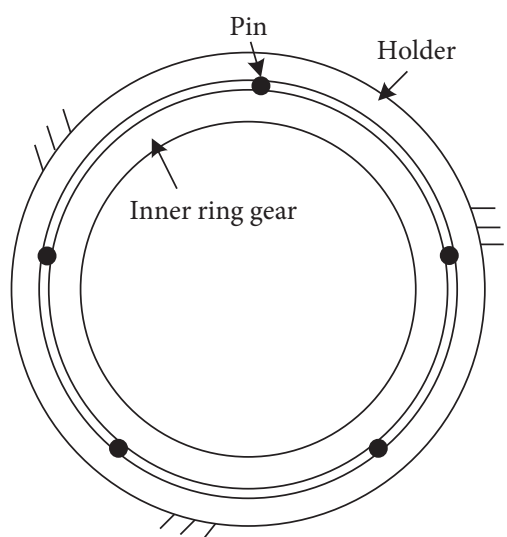

Figure 1: Schematic diagram of pin support in inner ring gear.

laying a foundation for the calculation of meshing stiffness of inner ring gear. The supporting mode of this paper is pin connection, and its boundary condition is the first mode according to the actual situation.

When calculating the influence of inner ring gear deformation on meshing stiffness, the inner gear teeth are treated as a rigid body, and the meshing force acts on the inner ring gear in the form of circumferential force, radial force, and torque. The relation is

$$
\left\{\begin{array}{l}
F_{r}=F_{n} \cos \alpha, \\
F_{t}=F_{n} \sin \alpha, \\
m_{0}=F_{t}|O B|-F_{r}|A B| .
\end{array}\right.
$$

The circumferential displacement, radial displacement, and bending angle of the inner ring gear are obtained by transforming the obtained circumferential force and radial force into distributed force, and the displacement of the contact point on the tooth profile caused by the inner ring gear deformation on the line of action is obtained as

$$
\sigma=\omega \cos \alpha+\mu \sin \alpha+\theta|O A| \sin \beta
$$

\section{Theory of Curved Beam}

Two fourth-order simultaneous differential equations are usually needed to solve the internal force deformation of a curved beam. In this case, a series of linear equations determined by boundary conditions are solved by solving multiple integral constants. This will make the calculation work complicated, and when the boundary conditions change and the load moves, the closed solution is used to solve the general differential equation to calculate the deformation and internal force of the curved beam.

3.1. Load and Torque Is Applied in a Concentrated Way. This study mainly focuses on the influence of the flexible inner ring gear on the inner gearing stiffness. The inner ring gear is simplified into a smooth ring. According to the support boundary condition, the ring is divided into several sections, each section is regarded as a uniformly curved 
beam, and the deformation of the whole ring can be solved according to the boundary condition. When an external force acts on the tooth profile along the line of action, the displacement of the meshing point caused by the deformation of the flexible ring on the line of action is superimposed with the displacement of the meshing point caused by the deformation of the tooth. The ratio of force to total displacement is the synthetical stiffness of flexible inner ring gear.

The expressions of strain, curvature, and torsion rate can be obtained by analyzing uniformly curved beams. In accordance with the principle of general elastomer material mechanics, the following basic differential equation can be established by linking the internal force and deformation of the section:

$$
\left\{\begin{array}{l}
M_{x}=-E I_{x}\left(\frac{d^{2} \omega}{d z^{2}}-\frac{\phi}{r}\right) \\
T=-E I_{\omega}\left(\frac{d^{3} \phi}{d z^{3}}+\frac{1}{r} \frac{d^{3} \omega}{d z^{3}}\right)+G I_{d}\left(\frac{d \phi}{d z}+\frac{1}{r} \frac{d \omega}{d z}\right) \\
\frac{d M}{d \varphi_{z}}=-T-r Q
\end{array}\right.
$$

where $A$ is the cross-sectional area of the beam; $E$ is the modulus of elasticity; $G$ is the shear modulus; $I_{x}$ and $I_{y}$ correspond to the bending moment of inertia about the $x$ and $y$ axes; $I_{d}$ is the torsional moment of inertia about the $z$ axis; $I_{\omega}$ is the warp constant of the beam; $Q$ is the shear.

Through the derivation and transformation of the above formula, the differential equation of bending and the corresponding expression of torsional angle and bending angle are obtained. According to formula (4), the relation between internal force and deformation of section is derived. The simple torsion theoretical equation with omitted section warping is established as follows (the shear deformation is minor, which is ignored here):

$$
\left\{\begin{array}{l}
\frac{d^{3} \omega}{d \varphi_{z}^{3}}+\frac{d \omega}{d \varphi_{z}}=\frac{r^{3}}{E I}((1+k) T+r Q) \\
\phi=\frac{1}{r} \frac{d^{2} \omega}{d \varphi_{z}^{2}}+\frac{r}{E I} M \\
\theta=\frac{d \omega}{d z}=\frac{1}{r} \frac{d \omega}{d \varphi_{z}}
\end{array}\right.
$$

where $z=r \varphi_{z}$ and $k=E I / G I_{d}$.

When the concentrated load and torque is applied to any position of the beam, the internal force of each section of the beam is divided into two parts.

As shown in Figure 2, when the concentrated load $P$ and concentrated torque $T$ act on any position $\varphi_{p}$ of the beam, the internal force of each section of the beam is determined by equations (5) and (6):

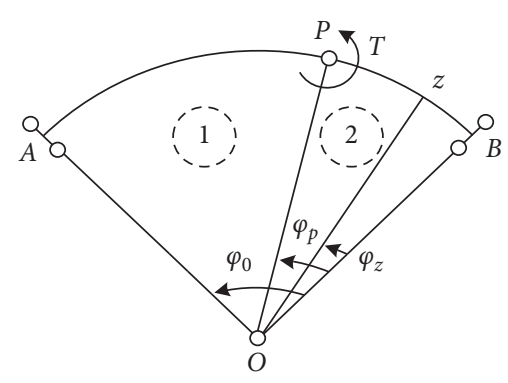

Figure 2: Calculation of deformation under concentrated load $P$ and concentrated torque $T$.

When $0 \leq \varphi_{z} \leq \varphi_{p}$,

$$
\left\{\begin{aligned}
M_{z p} & =(p r+T) \frac{\sin \left(\varphi_{0}-\varphi_{p}\right)}{\sin \varphi_{0}} \sin \varphi_{z}, \\
T_{z p} & =\operatorname{pr}\left[1-\frac{\varphi_{p}}{\varphi_{0}}-\frac{\sin \left(\varphi_{0}-\varphi_{p}\right)}{\sin \varphi_{0}} \cos \varphi_{z}\right] \\
- & T \frac{\sin \left(\varphi_{0}-\varphi_{p}\right)}{\sin \varphi_{0}} \cos \varphi_{z}, \\
Q_{z p} & =-p\left(1-\frac{\varphi_{p}}{\varphi_{0}}\right) .
\end{aligned}\right.
$$

When $\varphi_{p} \leq \varphi_{z} \leq \varphi_{0}$,

$$
\left\{\begin{aligned}
M_{z p} & =(p r+T) \frac{\sin \varphi_{p}}{\sin \varphi_{0}} \sin \left(\varphi_{0}-\varphi_{z}\right), \\
T_{z p} & =p r\left[\frac{\sin \varphi_{p}}{\sin \varphi_{0}} \sin \left(\varphi_{0}-\varphi_{z}\right)-\frac{\varphi_{p}}{\varphi_{0}}\right] \\
+ & T \frac{\sin \varphi_{p}}{\sin \varphi_{0}} \sin \left(\varphi_{0}-\varphi_{z}\right), \\
Q_{z p} & =p \frac{\varphi_{p}}{\varphi_{0}}
\end{aligned}\right.
$$

Taking the load point as the boundary, the curved beam is divided into two sections, $\varphi_{z}<\varphi_{p}$ and $\varphi_{z}>\varphi_{p}$, and the deflection differential equation obtained from above is

$$
\begin{gathered}
\frac{d^{3} \omega_{1}}{d \varphi_{z}^{3}}+\frac{d \omega_{1}}{d \varphi_{z}}=\frac{p r 3}{E I}\left\{\left[\left(1-\frac{\varphi_{p}}{\varphi_{0}}\right)-\frac{\sin \left(\varphi_{0}-\varphi_{p}\right)}{\sin \varphi_{p}} \cos \varphi_{z}\right]\right. \\
\left.-\left(1-\frac{\varphi_{p}}{\varphi_{0}}\right)\right\}-\frac{T r^{2}}{E I}(1+k) \frac{\sin \left(\varphi_{0}-\varphi_{p}\right)}{\sin \varphi_{p}} \cos \varphi_{z},
\end{gathered}
$$

$$
\begin{aligned}
& \frac{d^{3} \omega_{2}}{d \varphi_{z}^{3}}+\frac{d \omega_{2}}{d \varphi_{z}}=\frac{p r 3}{E I}\left\{(1+k) \frac{\sin \varphi_{p}}{\sin \varphi_{0}} \cos \left(\varphi_{0}-\varphi_{z}\right)-k \frac{\varphi_{p}}{\varphi_{0}}\right\} \\
& +\frac{T r^{2}}{E I}(1+k) \frac{\sin \varphi_{p}}{\sin \varphi_{0}} \cos \left(\varphi_{0}-\varphi_{z}\right) .
\end{aligned}
$$


The general solution of equations (7) and (8) is obtained as

$$
\left\{\begin{array}{l}
\omega_{1}=A+B \sin \varphi_{z}+C \cos \varphi_{z}+\frac{p r^{3}+T r^{2}}{E I} \\
* \frac{(1+k)}{2} \frac{\sin \left(\varphi_{0}-\varphi_{p}\right)}{\sin \varphi_{0}} \varphi_{z} \cos \varphi_{z}+\frac{p r^{3}}{E I} k\left(1-\frac{\varphi_{p}}{\varphi_{0}}\right) \varphi_{z} \\
\omega_{2}=D+E \sin \varphi_{z}+F \cos \varphi_{z}-\frac{p r^{3}+T r^{2}}{E I} \\
\quad \frac{(1+k)}{2} \frac{\sin \varphi_{p}}{\sin \varphi_{0}} \varphi_{z} \cos \left(\varphi_{0}-\varphi_{z}\right)-\frac{p r^{3}}{E I} k \frac{\varphi_{p}}{\varphi_{0}} \varphi_{z} .
\end{array}\right.
$$

The solution is based on the following boundary conditions and continuous conditions:

(1) $\varphi_{z}=0: \omega_{1}=0$ or $\omega_{1}^{\prime \prime}=0$

(2) $\varphi_{z}=\varphi_{p}: \omega_{1}=\omega_{2}, \omega_{1}^{\prime}=\omega_{2}^{\prime}$, and $\omega_{1}^{\prime \prime}=\omega_{2}^{\prime \prime}$

(3) $\varphi_{z}=\varphi_{p}: \omega_{2}=0$ and $\omega_{2}^{\prime \prime}=0$

The correlation coefficient is obtained by introducing boundary and continuous conditions:

$$
\left\{\begin{aligned}
A= & 0, C=0, \\
B= & \frac{p r^{3}+T r^{2}}{2 E I}(1+k) \frac{\varphi_{0} \sin \varphi_{p}-\varphi_{p} \cos \left(\varphi_{0}-\varphi_{p}\right) \sin \varphi_{p}}{\sin ^{2} \varphi_{p}} \\
& -\frac{p r^{3}}{2 E I}(1+3 k) \frac{\sin \left(\varphi_{0}-\varphi_{p}\right)}{\sin \varphi_{0}}-\frac{T r^{2}}{2 E I}(1+k) \frac{\sin \left(\varphi_{0}-\varphi_{p}\right)}{\sin \varphi_{0}} \\
D= & \frac{p r^{3}}{E I} k \varphi_{p}, \\
E= & \frac{p r^{3}}{2 E I}\left[(1+k) \frac{\varphi_{0} \sin \varphi_{p}}{\sin { }^{2} \varphi_{0}}-(1+k) \varphi_{p} \cos \varphi_{p} \cot \varphi_{0}\right. \\
& \left.+(1+3 k) \sin \varphi_{p} \cot \varphi_{0}\right] \\
F= & \frac{p^{3}}{2 E I}\left[(1+k) \varphi_{p} \cos \varphi_{p}-(1+3 k) \sin \varphi_{p}\right] \\
& +\frac{T^{2}}{2 E I}\left[\frac{(1+k)}{\tan \phi_{0}}\left(\frac{\varphi_{0} \sin \varphi_{p}}{\sin \varphi_{0} \cos \varphi_{0}}-\varphi_{p}+\sin \varphi_{p}\right)\right]
\end{aligned}\right.
$$

The obtained constants are substituted into the formula, and the bending of the curved beam under the action of concentrated load and concentrated torque and the corresponding formula of angle of bending and torsion are as follows:

When $0 \leq \varphi_{z} \leq \varphi_{p}$,

$$
\begin{aligned}
\omega_{1}= & \frac{\operatorname{pr} 3}{2 E I}\left\{\frac { ( 1 + k ) } { \operatorname { s i n } \varphi _ { 0 } } \left[\frac{\varphi_{0} \sin \varphi_{p}}{\sin \varphi_{0}} \sin \varphi_{z}-\varphi_{p} \cos \left(\varphi_{0}-\varphi_{p}\right) \sin \varphi_{z}\right.\right. \\
& \left.+\varphi_{z} \sin \left(\varphi_{0}-\varphi_{p}\right) \cos \varphi_{z}\right]-(1+3 k) \frac{\sin \left(\varphi_{0}-\varphi_{p}\right)}{\sin \varphi_{0}} \sin \varphi_{z} \\
& \left.+2 k\left(1-\frac{\varphi_{p}}{\varphi_{0}}\right) \varphi_{z}\right\}+\frac{\operatorname{Tr}^{2}}{2 E I}\left\{\frac { ( 1 + k ) } { \operatorname { s i n } \varphi _ { 0 } } \left[\frac{\varphi_{0} \sin \varphi_{p}}{\sin \varphi_{0}} \sin \varphi_{z}\right.\right.
\end{aligned}
$$$$
\left.-\varphi_{p} \cos \left(\varphi_{0}-\varphi_{p}\right) \sin \varphi_{z}+\varphi_{z} \sin \left(\varphi_{0}-\varphi_{p}\right) \cos \varphi_{z}\right]
$$$$
\left.-\sin \left(\varphi_{0}-\varphi_{p}\right) \cos \varphi_{z}\right\}
$$

$$
\theta_{1}=\frac{1}{r} \frac{d \omega_{1}}{d \varphi_{z}}
$$

$$
\begin{aligned}
= & \frac{p r^{2}}{2 E I}\left\{\frac { ( 1 + k ) } { \operatorname { s i n } \varphi _ { 0 } } \left[\frac{\varphi_{0} \sin \varphi_{p}}{\sin \varphi_{0}} \cos \varphi_{z}-\varphi_{p} \cos \left(\varphi_{0}-\varphi_{p}\right) \cos \varphi_{z}\right.\right. \\
& \left.-\varphi_{z} \sin \left(\varphi_{0}-\varphi_{p}\right) \sin \varphi_{z}\right]-2 k\left[\frac{\sin \left(\varphi_{0}-\varphi_{p}\right)}{\sin \varphi_{0}} \cos \varphi_{z}\right. \\
& \left.\left.-1+\frac{\varphi_{p}}{\varphi_{0}}\right]\right\}+\frac{\operatorname{Tr}}{E I}\left\{\frac { ( 1 + k ) } { 2 \operatorname { s i n } \varphi _ { 0 } } \left[\frac{\varphi_{0} \sin \varphi_{p}}{\sin \varphi_{0}} \cos \varphi_{z}\right.\right. \\
& \left.\left.-\varphi_{p} \cos \left(\varphi_{0}-\varphi_{p}\right) \cos \varphi_{z}-\varphi_{z} \sin \left(\varphi_{0}-\varphi_{p}\right) \sin \varphi_{z}\right]\right\},
\end{aligned}
$$

$$
\begin{aligned}
\varphi_{1}= & \frac{1}{r} \frac{d^{2} \omega_{1}}{d \varphi_{z}^{2}}+r \frac{M}{E I} \\
= & \frac{p r^{2}}{2 E I}\left\{\frac { ( 1 + k ) } { \operatorname { s i n } \varphi _ { 0 } } \left[\varphi_{p} \cos \left(\varphi_{0}-\varphi_{p}\right) \sin \varphi_{z}-\varphi_{0} \frac{\sin \varphi_{p}}{\sin \varphi_{0}} \sin \varphi_{z}\right.\right. \\
& \left.\left.-\sin \left(\varphi_{0}-\varphi_{p}\right)\left(\varphi_{z} \cos \varphi_{z}-\sin \varphi_{z}\right)\right]\right\} \\
& +\frac{\operatorname{Tr}}{E I}\left\{\frac { ( 1 + k ) } { 2 \operatorname { s i n } \varphi _ { 0 } } \left[\varphi_{p} \cos \left(\varphi_{0}-\varphi_{p}\right) \sin \varphi_{z}\right.\right. \\
& \left.-\varphi_{0} \frac{\sin \varphi_{p}}{\sin \varphi_{0}} \sin \varphi_{z}-\sin \left(\varphi_{0}-\varphi_{p}\right)\left(\varphi_{z} \cos \varphi_{z}-\sin \varphi_{z}\right)\right] \\
& \left.-k \frac{\sin \varphi_{p}}{\sin \varphi_{0}} \sin \left(\varphi_{0}-\varphi_{p}\right)\right\} .
\end{aligned}
$$




$$
\begin{aligned}
& \text { When } \phi_{p} \leq \phi_{z} \leq \phi_{0} \text {, } \\
& \omega_{2}=\frac{p r^{3}}{2 E I}\left\{\frac { ( 1 + k ) } { \operatorname { s i n } \varphi _ { 0 } } \left[\frac{\varphi_{0} \sin \varphi_{p}}{\sin \varphi_{0}} \sin \varphi_{z}+\varphi_{p} \sin \left(\varphi_{0}-\varphi_{p}\right) \cos \varphi_{p}\right.\right. \\
& \left.-\varphi_{z} \cos \left(\varphi_{0}-\varphi_{p}\right) \cos \varphi_{p}\right]-(1+3 k) \frac{\sin \left(\varphi_{0}-\varphi_{z}\right)}{\sin \varphi_{0}} \sin \varphi_{p} \\
& \left.+2 k \varphi_{p}\left(1-\frac{\varphi_{z}}{\varphi_{0}}\right)\right\}+\frac{\operatorname{Tr}^{2}}{E I}\left\{\frac { ( 1 + k ) } { 2 \operatorname { s i n } \varphi _ { 0 } } \left[\frac{\varphi_{0} \sin \varphi_{p}}{\sin \varphi_{0}} \sin \varphi_{z}\right.\right. \\
& +\varphi_{p} \sin \left(\varphi_{0}-\varphi_{p}\right) \cos \varphi_{p}-\varphi_{z} \cos \left(\varphi_{0}-\varphi_{p}\right) \sin \varphi_{p} \\
& \left.\left.-\sin \varphi_{p} \sin \left(\varphi_{0}-\varphi_{z}\right)\right]\right\} \\
& \theta_{2}=\frac{1}{r} \frac{d \omega_{2}}{d \varphi_{z}} \\
& =\frac{p r^{2}}{2 E I}\left\{\frac { ( 1 + k ) } { \operatorname { s i n } \varphi _ { 0 } } \left[\frac{\varphi_{0} \sin \varphi_{p}}{\sin \varphi_{0}} \cos \varphi_{z}-\varphi_{p} \cos \left(\varphi_{0}-\varphi_{z}\right) \cos \varphi_{p}\right.\right. \\
& \left.\left.-\varphi_{z} \sin \left(\varphi_{0}-\varphi_{z}\right) \sin \varphi_{p}\right]+2 k\left[\frac{\cos \left(\varphi_{0}-\varphi_{z}\right)}{\sin \varphi_{0}} \sin \varphi_{p}-\frac{\varphi_{p}}{\varphi_{0}}\right]\right\} \\
& +\frac{\operatorname{Tr}}{E I}\left\{\frac { ( 1 + k ) } { 2 \operatorname { s i n } \varphi _ { 0 } } \left[\frac{\varphi_{0} \sin \varphi_{p}}{\sin \varphi_{0}} \cos \varphi_{z}-\varphi_{p} \cos \left(\varphi_{0}-\varphi_{z}\right) \cos \varphi_{p}\right.\right. \\
& \left.\left.-\varphi_{z} \sin \left(\varphi_{0}-\varphi_{z}\right) \sin \varphi_{p}\right]\right\} \\
& \varphi_{2}=\frac{1}{r} \frac{d^{2} \omega_{2}}{d \phi_{z}^{2}}+r \frac{M}{E I} \\
& =\frac{p r^{2}}{2 E I}\left\{\frac { ( 1 + k ) } { \operatorname { s i n } \varphi _ { 0 } } \left[\varphi_{z} \cos \left(\varphi_{0}-\varphi_{z}\right) \sin \varphi_{p}-\varphi_{0} \frac{\sin \varphi_{p}}{\sin \varphi_{0}} \sin \phi_{z}\right.\right. \\
& \left.\left.-\sin \left(\varphi_{0}-\varphi_{z}\right)\left(\varphi_{p} \cos \varphi_{p}-\sin \varphi_{p}\right)\right]\right\} \\
& +\frac{p r^{2}}{2 E I}\left\{\frac { ( 1 + k ) } { \operatorname { s i n } \varphi _ { 0 } } \left[\varphi_{z} \cos \left(\varphi_{0}-\varphi_{z}\right) \sin \varphi_{p}-\varphi_{0} \frac{\sin \varphi_{p}}{\sin \varphi_{0}} \sin \varphi_{z}\right.\right. \\
& \left.\left.-\sin \left(\varphi_{0}-\varphi_{z}\right)\left(\varphi_{p} \cos \varphi_{p}-\sin \varphi_{p}\right)\right]\right\} \\
& +\frac{\operatorname{Tr}}{E I}\left[\frac { ( 1 + k ) } { 2 \operatorname { s i n } \varphi _ { 0 } } \left[\varphi_{z} \cos \left(\varphi_{0}-\varphi_{z}\right) \sin \varphi_{p}-\varphi_{0} \frac{\sin \varphi_{p}}{\sin \varphi_{0}} \sin \varphi_{z}\right.\right. \\
& \left.-\sin \left(\varphi_{0}-\varphi_{z}\right)\left(\varphi_{p} \cos \varphi_{p}-\sin \varphi_{p}\right)\right] \\
& \left.-k \frac{\sin \left(\varphi_{0}-\varphi_{z}\right)}{\sin \varphi_{0}} \sin \varphi_{p}\right\}
\end{aligned}
$$

By establishing the above theoretical analytical formulas, the deformation of curved beams under different loads at different positions was calculated easily and quickly, which provided an important theoretical basis for the relevant theoretical analysis. At the same time, it also provides important theoretical support for the solution of the influence of flexible inner ring gear on the synthetical stiffness.

\subsection{Comparison and Verification with Finite Element Method.} In the finite element software ABAQUS, the circular curved beam structure was established, and the density of the model was set as $7.85 e-9 \mathrm{~kg} / \mathrm{mm}^{3}$, the elastic modulus was $2.06 e+5 \mathrm{GPa}$, and the shear modulus was $7.938 e+4 \mathrm{GPa}$. The beam element type B21 is adopted, and the section shape and size of the beam is set (rectangular section with thickness of $20 \mathrm{~mm}$ and tooth width of $50 \mathrm{~mm}$ ). At the same time, it is divided into $n$ sections ( 5 sections in this paper), and different coordinate systems are set at the splitting points, so as to set different boundary conditions through the splitting points. According to the above, its deformation in different directions is obtained, and its radial displacement (bending), bending angle, and torsion angle are obtained. There is an order of magnitude difference between shear deformation and deflection bending due to shear. Therefore, we can ignore its influence and obtain the following theory and finite element comparison.

It can be seen from the comparison of the above Figures 3 and 4 that the formula based on the structural mechanics theory and the finite element model are highly consistent with the bending and bending angle of the curved beam. It is verified that the formula established in the theoretical analysis is reasonable and accurate, which can quickly and accurately obtain the bending of the curved beam, thus providing a solid theoretical foundation for calculating the flexible deformation of the inner ring gear. Meanwhile, the stress and deformation of the inner ring gear are analyzed in the first part of this paper. The related displacement derived from the theory and the transfer of meshing position caused by the bending angle of the inner ring gear circumferential are combined. The comprehensive deformation of the gear teeth caused by the flexibility of the inner ring gear is obtained, as shown in Figure 5.

\section{Calculation of Inner Gearing Meshing Stiffness}

The synthetical stiffness $k$ of inner gearing can be divided into two parts, namely, the stiffness $k_{\mathrm{r}}$ caused by the flexibility of the inner ring gear and the meshing stiffness $k_{\mathrm{c}}$ of the gear tooth of inner gearing (without considering the flexibility of the inner ring gear). There are two methods to calculate the time-varying meshing stiffness $k_{\mathrm{c}}$ of the gear tooth of inner gearing [11]. Each one applies to a different situation. The LTCA method adopted in this paper can accurately obtain the meshing stiffness of gears. The LTCA method is used to calculate the normal displacement of each instantaneous discrete point after tooth deformation. At the 


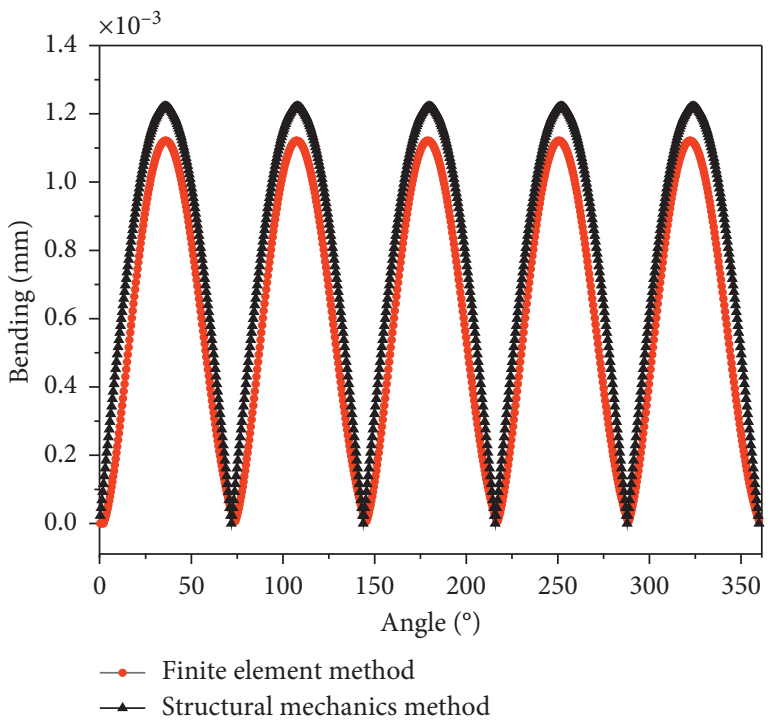

FIgURE 3: Comparison of bending of curved beams by two methods.

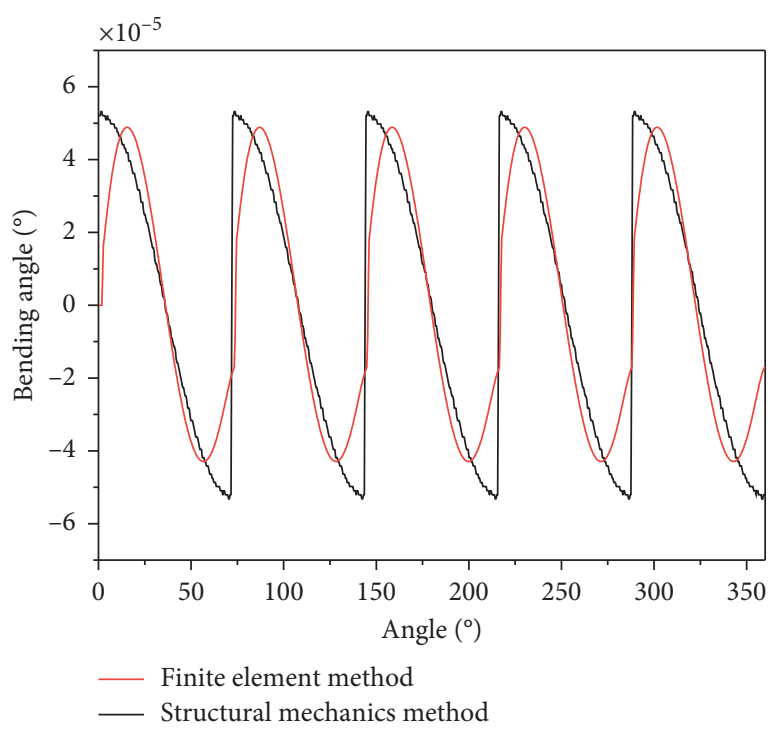

Figure 4: Comparison of bending angle of curved beam by two methods.

same time, the method solves the discrete load at the discrete point of the instantaneous contact ellipse long axis and the normal clearance of the tooth surface at the discrete point of the instantaneous contact ellipse long axis after the tooth deformation. This method can accurately obtain the discrete time-varying meshing stiffness of gears at different meshing points, which is similar to the method of local slope mesh stiffness proposed by Cooley et al. [11]. The LTCA method is suitable for the calculation of the planetary transmission system. Table 1 is the helical gear parameters.

In this paper, LTCA (load tooth contact analysis) [12] is used to calculate the total meshing stiffness of gear teeth considering the installation error. The basic idea of gear

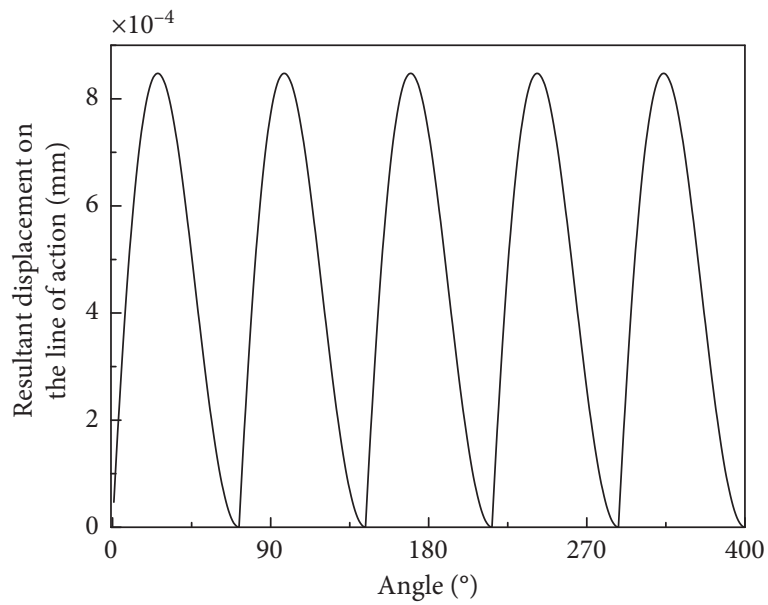

FIgURE 5: The overall deformation of the flexible inner ring gear is on the line of action.

TABLE 1: Helical gear parameters.

\begin{tabular}{|c|c|c|c|}
\hline Parameters & Unit & Driving pinion & Driven gear \\
\hline Tooth number, $Z_{1}$ and $Z_{2}$ & - & 20 & 80 \\
\hline Normal module, $m_{n}$ & $\mathrm{~mm}$ & 5 & 5 \\
\hline Normal pressure angle, $\alpha_{n}$ & deg & 20 & 20 \\
\hline Helix angle, $\beta_{m}$ & deg & 20 & 20 \\
\hline Face width, $B$ & $\mathrm{~mm}$ & 50 & 50 \\
\hline Addendum, $h_{a}$ & $\mathrm{~mm}$ & 5 & 5 \\
\hline Dedendum, $h_{f}$ & $\mathrm{~mm}$ & 6.75 & 6.75 \\
\hline Elastic modulus, $E$ & $\mathrm{GPa}$ & \multicolumn{2}{|l|}{210} \\
\hline Density, $\rho$ & $\mathrm{g} / \mathrm{cm}^{3}$ & \multicolumn{2}{|c|}{7.85} \\
\hline Speed, $v$ & $\mathrm{r} / \mathrm{min}$ & \multicolumn{2}{|c|}{1500} \\
\hline Power & $\mathrm{kw}$ & \multicolumn{2}{|c|}{400} \\
\hline
\end{tabular}

tooth meshing stiffness calculation is through TCA (tooth contact analysis) $[13,14]$ and LTCA (load tooth contact analysis). By using the deformation coordination equation, force balance, and nonembedding conditions, the total normal contact deformation of the meshing tooth surface is obtained, and the comprehensive meshing stiffness of the gear tooth is obtained by conversion. Considering the gear tooth, surface error resulted in the manufacturing process of impact load gear shaft torsional deformation and additional flexibility matrix effect on tooth surface composite flexibility matrix and the installation error and the effects of tooth surface modification, thus can accurately calculate the tooth mesh stiffness. Figure 6 is the flow chart based on the method of LTCA to calculate the integrated meshing stiffness.

4.1. Deformation Coordination Equation. Suppose there are two pairs of gear tooth I and II meshing at the same time. The load tooth contact analysis model shown in Figure 7 shows the cross section of the tooth flank along the long axis of the contact ellipse. $i$ is the center of instantaneous contact ellipse on the tooth pair, $j$ is the discrete point along the long axis on the tooth pair, and $w$ is the initial pitch before contact on the tooth pair. 


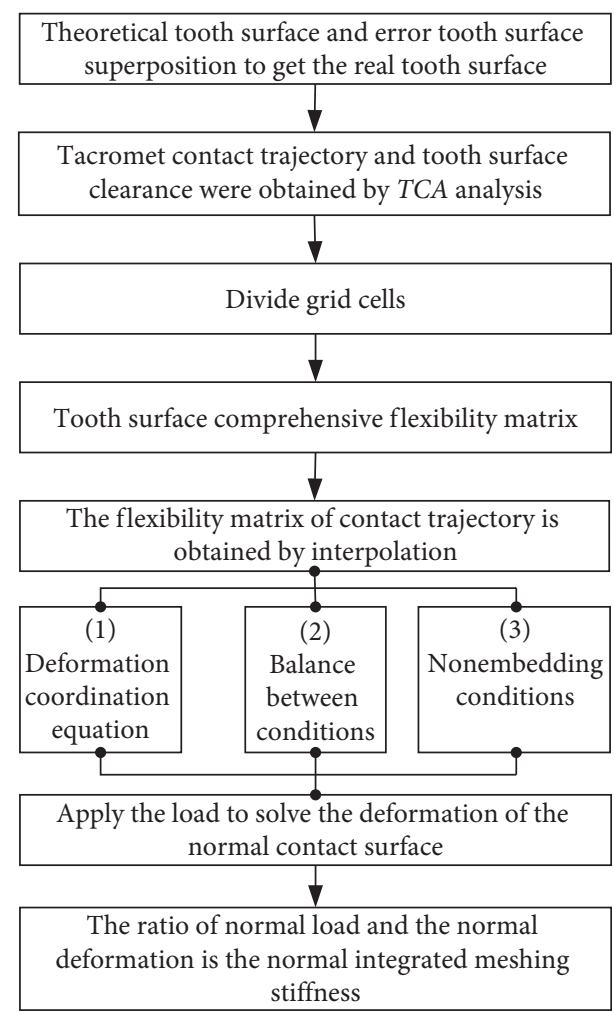

FIGURE 6: Flow chart for calculating the integrated meshing stiffness.

Before gear deformation, the teeth of I and II tooth surface initial clearance are

$$
\begin{aligned}
{[w]_{k} } & =[\delta]_{k}+[b]_{k}, \quad k=\mathrm{I}, \mathrm{II}, \\
{[w]_{k} } & =\left[w_{1}, w_{2}, \ldots, w_{i}, \ldots, w_{n}\right]^{T}, \\
{[b]_{k} } & =\left[b_{1}, b_{2}, \ldots, b_{i}, \ldots, b_{n}\right]^{T}, \\
{[\delta]_{k} } & =\delta[1,1, \ldots, 1, \ldots, 1]^{T}, \\
b_{M_{0}} & =\sqrt{\left(x_{1}-x_{2}\right)^{2}+\left(y_{1}-y_{2}\right)^{2}+\left(z_{1}-z_{2}\right)^{2}},
\end{aligned}
$$

where $n$ is the number of discrete points on the long axis of the instantaneous contact ellipse. $b_{j}(j=1,2, \ldots, n)$ is the tooth surface normal clearance at the discrete point $j$ of the long axis of the instantaneous contact ellipse. $\delta$ is the transmission error of gear pair $k$. Instantaneous contact ellipse center $b_{i}=0$. The clearance $b_{j}(j \neq i)$ at other discrete points along the major axis of the ellipse is given by (17) [12]. $b_{M_{0}}$ is the normal clearance between the two teeth contacts.

Under the action of discrete load $P$, the gear teeth are elastically deformed. If the driving pinion is fixed and the driven gear tooth moves along the normal direction under load, the displacement coordination equation is [15]

$$
[F]_{k}[p]_{k}+[w]_{k}=[Z]+[d]_{k}, \quad k=\mathrm{I}, \mathrm{II} .
$$

Among them,

$$
\begin{aligned}
{[p]_{k} } & =\left[p_{1}, p_{2}, \ldots, p_{i}, \ldots, p_{n}\right]^{T}, \\
{[d]_{k} } & =\left[d_{1}, d_{2}, \ldots, d_{i}, \ldots, d_{n}\right]^{T}, \\
{[Z]_{k} } & =Z[1,1, \ldots, 1, \ldots, 1]^{T},
\end{aligned}
$$

where $p_{j}(j=1,2, \ldots, n)$ is the normal load at the discrete point $j$ of the long axis of instantaneous contact ellipse of tooth pair $k . d_{j}(j=1,2, \ldots, n)$ is the deformed tooth surface clearance at the discrete point $j$ of the instantaneous contact ellipsoid of tooth pair $k$. $Z$ is the normal displacement of gear teeth. $[F]_{k}$ is the normal synthetic flexibility matrix of tooth pair $k$.

4.2. Force Balance Conditions. Discrete loads $p_{j}(j=1,2, \ldots$, $n)$ are satisfied as follows:

$$
\sum_{j=1}^{n} p_{j \mathrm{I}}+\sum_{j=1}^{n} p_{j \mathrm{II}}=P
$$

\subsection{Nonembedding Conditions}

$$
\begin{aligned}
& \text { If } p_{j k}>0 \text {, then } d_{j k}=0, \\
& \text { if } p_{j k}=0 \text {, then } d_{j k}>0 .
\end{aligned}
$$

where $k=\mathrm{I}$, II.

4.4. Loaded Tooth Contact Analysis. The contact problem of loaded gear teeth was solved by mathematical programming:

$$
\left\{\begin{array}{l}
f=\min \sum_{j=1}^{2 n+1} X_{j}, \\
-[F][p]+[Z]+[d]+[X]=[w], \\
P=[e]^{T}[p]+X_{2 n+1},
\end{array}\right.
$$

$$
\text { S.t. } p_{j} \geq 0, d_{j} \geq 0, Z \geq 0, X_{j} \geq 0, \quad p_{j}=0 \text { or } d_{j}=0 \text {, }
$$

where $X_{j}(j=1,2, \ldots, 2 n)$ is the artificial variable. $[X]=\left[X_{1}\right.$, $\left.X_{2}, \ldots, X_{2 n}\right]^{T}$. Each element of the $n$-dimensional column vector $[\mathrm{e}]$ is 1 .

Solving equations (22) and (23) give the normal displacement $Z$ after gear teeth deformation and the discrete load $[p]$ at the discrete points of the long axis of the instantaneous contact ellipse. At the same time, the tooth surface normal clearance $[d]$ at instantaneous contact ellipse long axis discrete point is obtained after gear teeth deformation.

The transmission error of gear pair under load can be obtained by converting the normal displacement $Z$ of gear tooth deformation into angle value. From the discrete load $[p]$ at discrete points along the long axis of the instantaneous contact ellipse, the distribution coefficients of the loads I and II at a certain contact position are given as follows: 


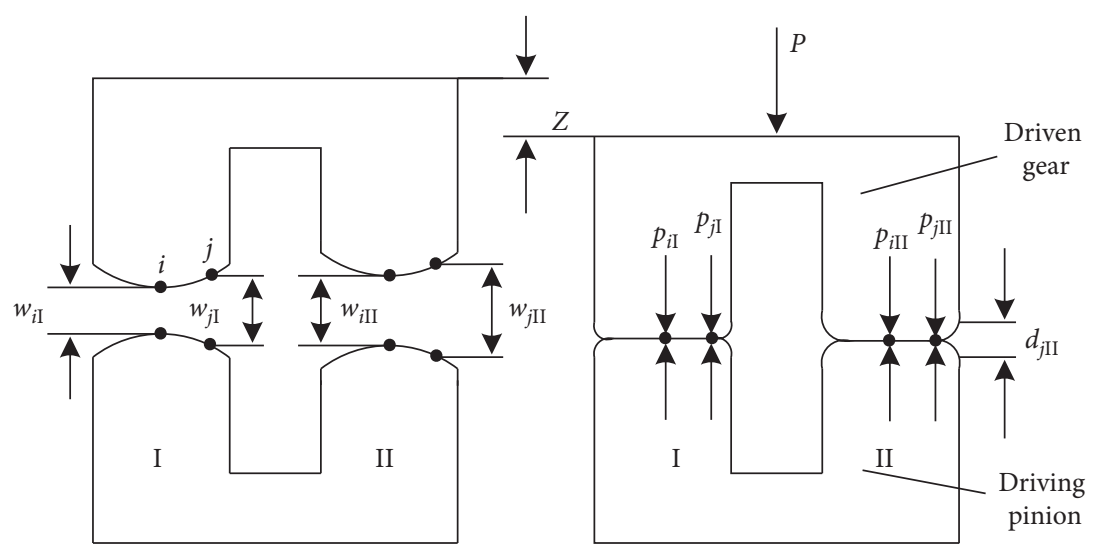

FIgURE 7: The model of helical gear LTCA.

$$
L_{k}=\frac{\sum_{j=1}^{n} p_{j k}}{P}, \quad k=\mathrm{I}, \mathrm{II} .
$$

If $L_{k}$ is less than 1 , the current contact position for more pairs of gear meshing.

The synthetical time-varying meshing stiffness of the inner gearing teeth is shown in Figure 8.

Through the comprehensive deformation amount on the line of action caused by the flexible inner ring gear, the deformation stiffness of the flexible inner ring gear is obtained as shown in Figure 9. It can be seen from Figure 9 that the stiffness at the positions of the support points will increase significantly, which is consistent with the actual situation. At the same time, the synthetical time-varying meshing stiffness of inner gear in the meshing process is composed of two parts, namely, meshing stiffness generated by tooth deformation and deformation stiffness of flexible inner ring gear. After the normal meshing force of the tooth is decomposed (see Figure 10), the stiffness under different resolution is obtained. The synthetical meshing stiffness of inner gearing is shown in Figure 11.

As shown in Figure 11, the synthetical meshing stiffness of inner gearing is shown in Figure 11, where Figure 11(b) is the partial magnification of Figure 11(a). It can be seen from Figure 11 that the time-varying meshing stiffness of the gear teeth formed small fluctuations on the deformation stiffness of the flexible inner ring gear, and the overall comprehensive meshing stiffness was complex. The detailed analysis in this paper laid a very important foundation for the later study on the dynamics of the planetary transmission system. In this paper, five supporting points are used for the flexible inner ring gear (corresponding modifications can be made according to the actual supporting points). The selection of five supporting points here is to make it more convenient and obvious to see the overall impact of the flexibility of the inner ring gear on the meshing stiffness. By combining the geometrical structure of the inner ring gear, the whole inner ring gear is divided into five segments of the curved beam, and a comprehensive internal mesh stiffness model with wheel tooth deformation and ring gear deformation is established by combining the curved beam theory. It lays an important theoretical foundation for the analysis of the gear transmission process.

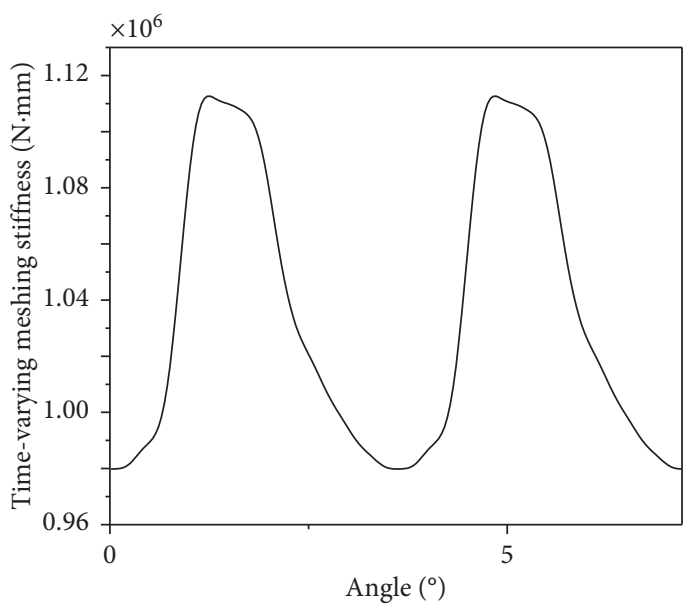

Figure 8: The synthetical time-varying meshing stiffness of the inner gearing teeth.

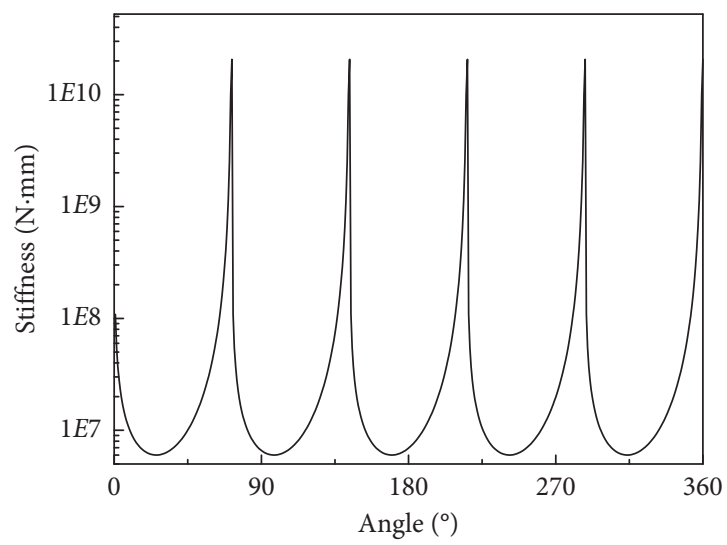

FIGURE 9: The meshing stiffness caused by the flexibility of the inner ring gear.

\section{Conclusion}

By segmenting the inner ring gear into a curved beam, an algorithm and a model for calculating the meshing stiffness of the flexible inner ring gear are established. In the model, the effects of the parameters such as the 


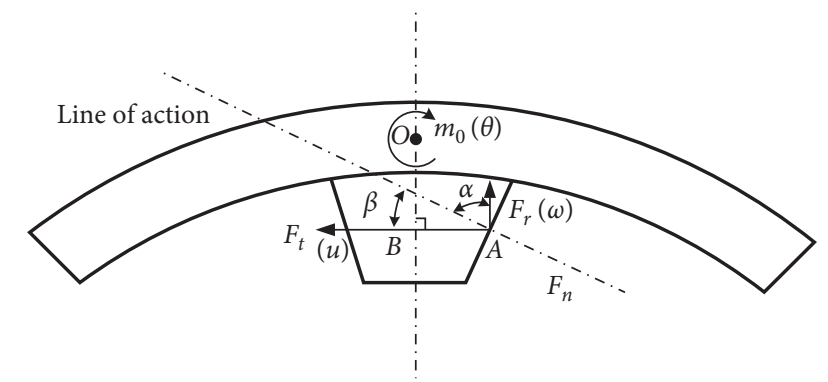

FIGURE 10: Displacement of inner ring gear deformation along the line of action (including force decomposition).

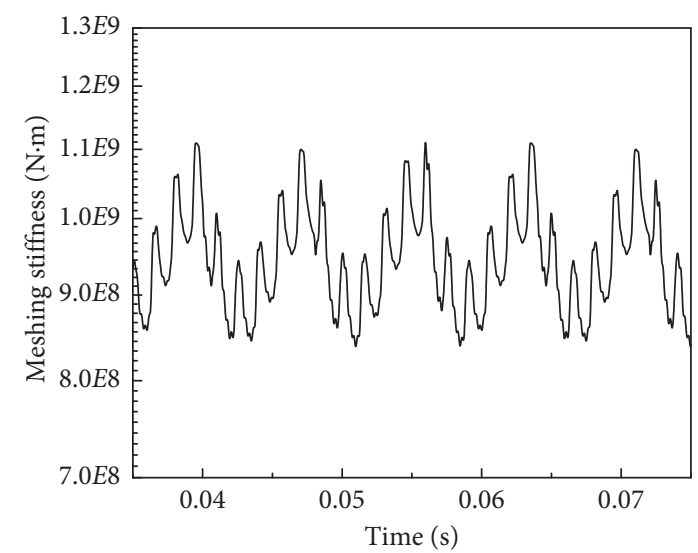

(a)

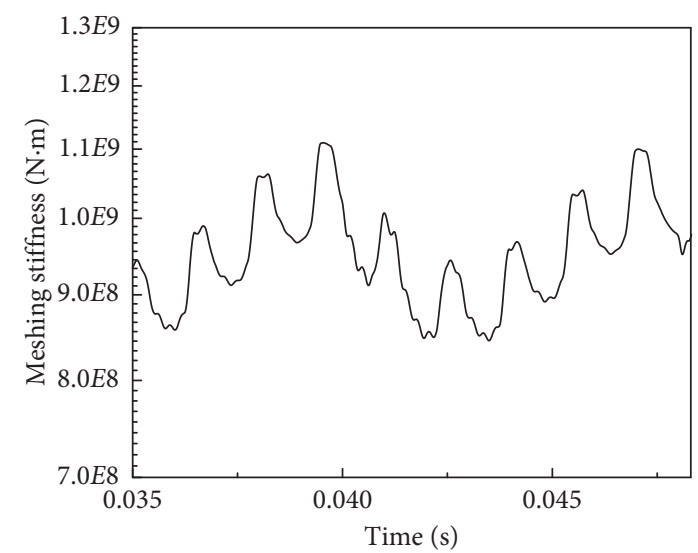

(b)

FIGURE 11: The synthetical meshing stiffness of inner gearing: (a) full circumference and (b) one-third.

supporting mode, supporting quantity, the thickness, and shape of the sections about the meshing stiffness of the planetary gear transmission system are considered comprehensively. At the same time, the comparison with the finite element model verifies the accuracy and reliability of the method. This study provides the basis and method for the optimization design of the flexible inner ring gear of planetary gear transmission, which is of great engineering significance. It also provides a more accurate model and foundation for studying the dynamic performance of the planetary transmission system.

\section{Data Availability}

The data used to support the findings of this study are available from the corresponding author upon request.

\section{Conflicts of Interest}

The authors declare that they have no conflicts of interest.

\section{Acknowledgments}

This work was supported by the National Natural Science Foundation of China (51375384).

\section{References}

[1] A. Kahraman and S. Vijayakar, "Effect of internal gear flexibility on the quasi-static behavior of a planetary gear set," Journal of Mechanical Design, vol. 123, no. 3, pp. 408-415, 2001.

[2] A. Kahraman, A. A. Kharazi, and M. Umrani, "A deformable body dynamic analysis of planetary gears with thin rims," Journal of Sound and Vibration, vol. 262, no. 3, pp. 752-768, 2003.

[3] X. Wu and R. G. Parker, "Vibration of rings on a general elastic foundation," Journal of Sound and Vibration, vol. 295, no. 1-2, pp. 194-213, 2006.

[4] J. Zhang, Y. M. Song, and J. J. Wang, "Dynamic modeling for spur planetary gear transmission with flexible ring gear," Journal of Mechanical Engineering, vol. 45, no. 12, pp. 29-36, 2009.

[5] J. Zhang, X. Z. Liu, and Y. Jiao, "Vibration analysis of planetary gear trains based on a discrete-continuum dynamic model," Journal of Mechanical Engineering, vol. 50, no. 15, pp. 104-112, 2014.

[6] H. C. Xu and D. T. Qin, "Vibration response of flexible spur ring gear with elastic foundation under internal excitation," Journal of Mechanical Engineering, vol. 54, no. 9, pp. 161-167, 2018.

[7] L. X. Wang, S. M. Wu, and J. H. Liu, "Dynamic characteristics analysis of the planetary gear system with the flexible ring," Mechanical design and manufacture, vol. 1, pp. 40-43, 2018.

[8] Z. G. Chen, Study on Gear Mesh Nonlinear Excitation Modeling and Vibration Features of Planetary Gear System, pp. 48-53, University of Chongqing, Chongqing, China, 2013.

[9] Z. Chen, Y. Shao, and D. Su, "Dynamic simulation of planetary gear set with flexible spur ring gear," Journal of Sound and Vibration, vol. 332, no. 26, pp. 7191-7204, 2013.

[10] L. S. Yao, Curved Beam, pp. 67-80, People's Communications Press, Beijing, China, 1989.

[11] C. G. Cooley, C. Liu, X. Dai, and R. G. Parker, "Gear tooth mesh stiffness: a comparison of calculation approaches," Mechanism and Machine Theory, vol. 105, pp. 540-553, 2016.

[12] Z. D. Fang, "Models and methods for loaded gear tooth contact analysis (LTCA)," Journal of Mechanical Transmission, vol. 22, no. 2, pp. 1-3, 1998.

[13] F. L. Litvin and A. Fuentes, Gear Geometry and Applied Theory, Cambridge University Press, Cambridge, UK, 2004.

[14] J. Argyris, A. Fuentes, and F. L. Litvin, "Computerized integrated approach for design and stress analysis of spiral bevel 
gears," Computer Methods in Applied Mechanics and Engineering, vol. 191, no. 11-12, pp. 1057-1095, 2002.

[15] J. Wang, F. B. Oswald, and J. J. Coy, Effect of Extended Tooth Contact on the Modeling of Spur Gear Transmissions, National Aeronautics and Space Administration, Washington, DC, USA, 1993. 


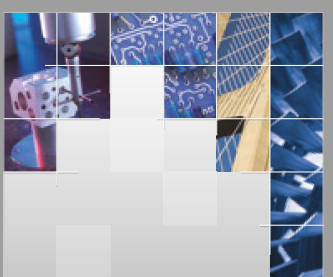

\section{Enfincering}
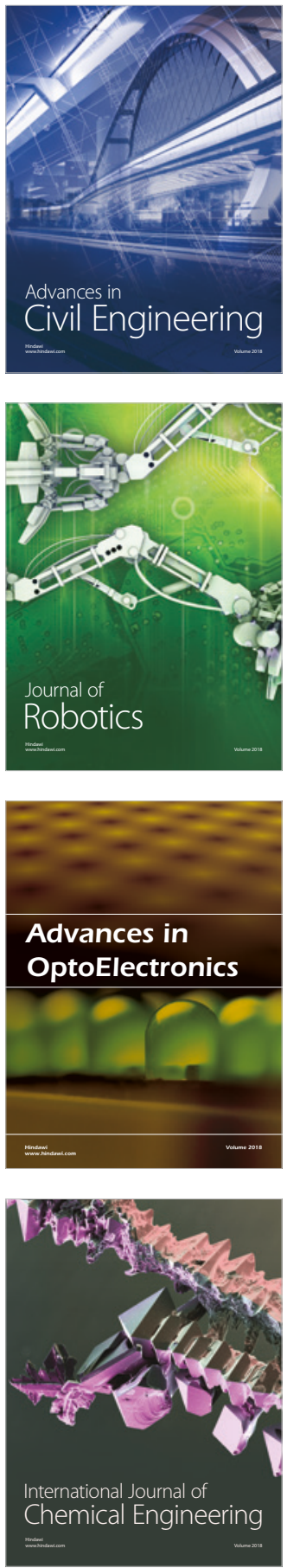

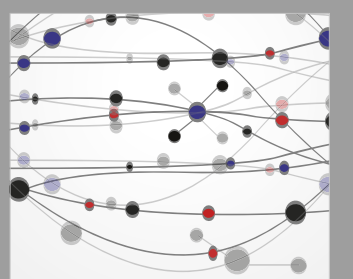

\section{Rotating \\ Machinery}

The Scientific World Journal

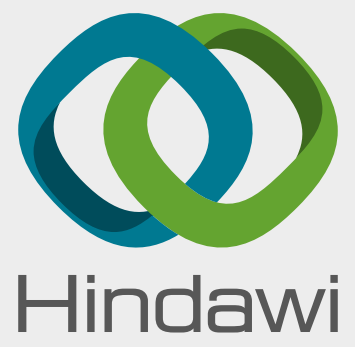

Submit your manuscripts at

www.hindawi.com
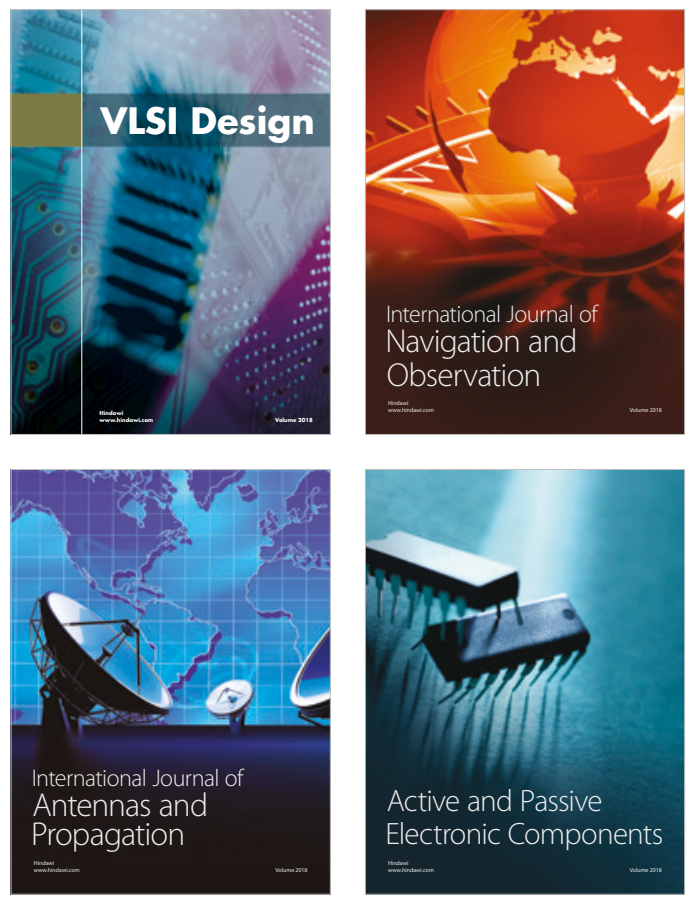
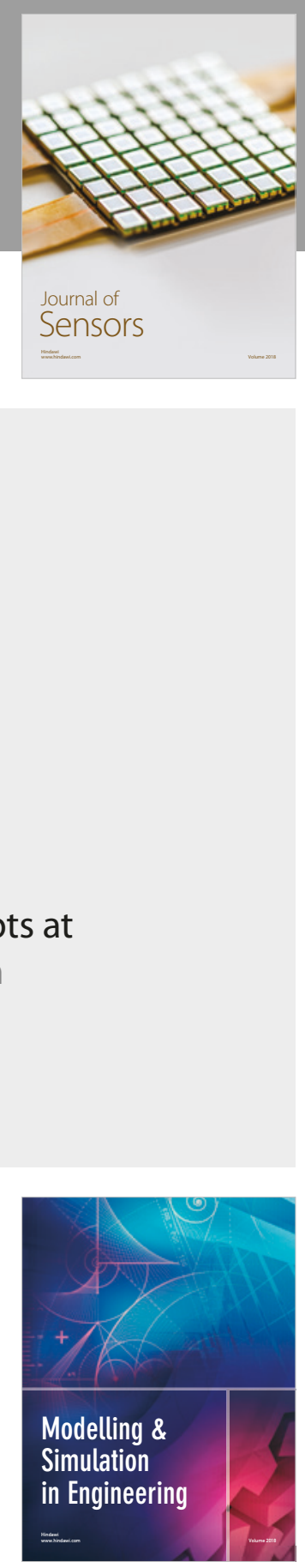

\section{Advances \\ Multimedia}
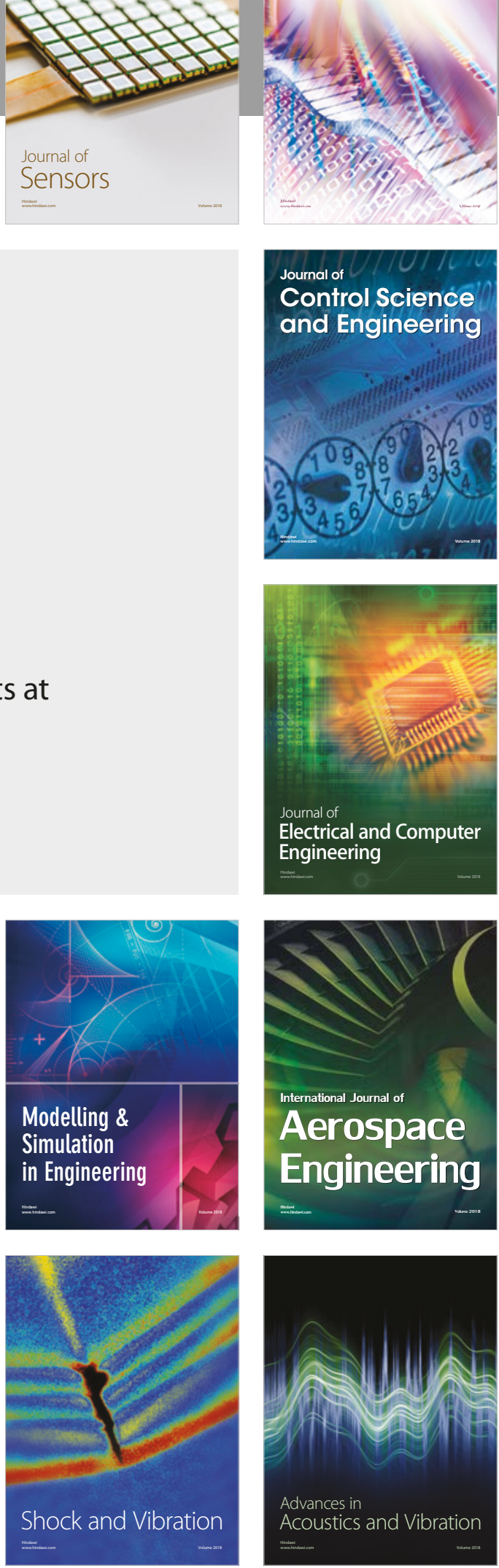